\title{
Tyrosine phosphorylation as a signaling component for plant improvement
}

\author{
Youn-Il Park $\cdot$ Hyo-Sik Yang $\cdot$ Man-Ho Oh
}

Received: 27 October 2015 / Revised: 7 November 2015 / Accepted: 7 November 2015

(C) Korean Society for Plant Biotechnology

\begin{abstract}
Plant genome analyses, including Arabidopsis thaliana showed a large gene family of plant receptor kinases with various extracellular ligand-binding domain. Now intensively studies to understand physiological and cellular functions for higher plant receptor kinases in diverse and complex biological processes including plant growth, development, ligands perception including steroid hormone and plant-microbe interactions. Brassinosteroids (BRs) as a one of well know steroid hormone are plant growth hormones that control biomass accumulation and also tolerance to many biotic and abiotic stress conditions and hence are of relevance to agriculture. BRI1 receptor kinase, which is localized in plasma membrane in the cell sense BRs and it bind to a receptor protein known as BRASSINOSTEROIDINSENSITIVE 1 (BRI1). Recently, we reported that BRI1 and its co-receptor, BRI1-ASSOCIATED KINASE (BAK1) autophosphorylated on tyrosine residue (s) in vitro and in vivo and thus are dual-specificity kinases. Other plant receptor kinases are also phosphorylated on tyrosine residue (s). Post-translational modifications (PTMs) can be studied by altering the residue modified by directed mutagenesis to mimic the modified state or to prevent the modification. These approaches are useful to not only characterize the regulatory role of a given modification, but may also provide opportunities for plant improvement.
\end{abstract}

Keywords Post-translational modification, Brassinosteroids, BRASSINOSTEROIDINSENSITIVE 1, Plant Receptor Kinases, BRI1-ASSOCIATED KINASE1, Tyrosine phosphorylation

Y.-I. Park $\cdot$ M.-H. Oh $(\bowtie)$

Department of Biological Sciences, Chungnam National

University, Daejeon 34134, Korea

e-mail: manhooh@cnu.ac.kr

H.-S. Yang

Department of Biology, College of Natural Science, Mokpo

National University, Muan-gun Jeonnam, Korea

\section{Introduction}

Receptor-like kinases (RLKs) of Arabidopsis thaliana showed a large gene family of about 600 genes that containing about $2 \%$ of protein-coding genes. These higher plant RLKs have an organization of functional importance domains similar to that of animal RLKs, which consisted of extracellular domain, transmembrane domain and an intracellular cytoplasmic domain. Generally, the ability to recognize and transduce signals from specific molecules via cell surface receptors is basic property of all living systems. The family of receptor tyrosine kinases (RTKs) mediates many interesting signaling events such as proliferation, differentiation, and apoptosis at the cell surface in animal system. As we know, plant RLKs are generally classified as serine/threonine kinases. However, several research group reported that plant RLKs were involved in the tightly control of growth, plant morphogenesis, diverse pathogen resistance, and responses to biotic and abiotic stresses (Becraft, 2002; de Lorenzo et al. 2009; Tor et al. 2009, Torii, 2004), but the functions of most plant RLKs and important family of signal transduction molecules still unknown. Among the Arabidopsis RLKs, this family can be divided into over 20 families based on the structure of the extracellular ligand-binding domain, with Leucine-Rich Repeats (LRRs). The most well-characterized LRR-RLKs, which is BRI1 and BAK1, both protein kinases critically involved in brassinosteroid signaling pathway (Clouse, 2002; Gendron et al. 2007; Vert et al. 2005); CLAVATA1, controlling shoot apical meristem cell fate (DeYoung et al. 2006), ERECTA, stomata development and patterning (Torii, 2004); HAESA, which plays a role in floral abscission (Cho et al. 2008); FLS2, capable of binding the bacterial flagellin peptide ligand (Boller and Felix, 2009); and AtSERK1, involved in somatic embryogenesis (Nodine et al. 2007); VH1 (VASCULAR HIGHWAY1) influencing leaf cell patterning (Nicole and Nelson, 2002) and EMS1 (EXCESS MICROSPOROCYTES 1) which plays an important role in microsporogenesis and male sterility (Zhao et al. 2002). In no case, however, has a complete comparative analysis of biochemical properties between kinases of different subfamilies been undertaken.

Interestingly, the molecular mechanism of animal RLKs is 
Table 1 Alignment of subdomain VIb of receptor kinases for seven RD-type and two non-RD-type (FLS2 and SRF9) receptor kinases

\begin{tabular}{|c|c|c|}
\hline Name & Locus (AGI) & Subdomain VIb sequence \\
\hline REPR1 & At1g73080 & PIVHRDIKPE \\
\hline SOBIR1 & At 231880 & RIIHRDLKPA \\
\hline PSKR1 & At2g02220 & PHILHRDIKSS \\
\hline PSYR1 & Atlg72300 & PHIVHRDIKSS \\
\hline HAESA & At4g28490 & PIVH $\underline{R D V K S S}$ \\
\hline SRF9 & Atlg11130 & PVVHQNFKSS \\
\hline FLS2 & At5g20480 & PIVHCDLKPA \\
\hline BRI1 & At4g39400 & HIIHRDMKSS \\
\hline BAK1 & At4g33430 & KIIHRDVKAA \\
\hline
\end{tabular}

very well characterized and involves specific ligand-mediated dimerization (homo-and hetero-) of the receptor kinases followed by phosphorylation of the intracellular cytoplasmic domain of receptor kinases. In the presence of ligands, activation of receptor kinases results in recognition and transphosphorylation of downstream components, leading eventually to regulation in specific gene expression and/or non-genomic changes in cellular physiology (Heldin, 1995). To thoroughly analyze higher plant LRR-RLK function, it is an importance to understand the role of receptor dimerization including homo-and heterodimerization and phosphorylation of cytoplasmic domain, including identification of specific phosphorylation sites, their functional significance and identification of substrates for LRR-RLKs. Among LRR-RLKs, the most thoroughly studied plant LRR-RLKs are BRI1, and its co-receptor, BAK1 in BR signaling (Vert et al. 2005). As we shown on Table 1, both BRI1 and BAK1 belong to the RD group of kinases, but, FLS2 and EFR belong to the non-RD group of receptor kinases and are important for anti-bacterial immunity (Boller and Felix, 2009). However, recent report suggested that some of LRR-RLKs and calcium-dependent protein kinase (CDPK) are dual-specificity kinases that can autophosphorylates on serine, threonine, and tyrosine residues (Karlova et al. 2009; Oh et al. 2009; 2010; 2012).

In terms of BR signaling after binding of BR to BRI1 receptor kinase, Wang et al. (2005) reported that BRI1 and BAK1 activation in vivo follows a sequential transphosphorylation model in which BRI1 regulates signaling specificity by directly binding the hormone followed by some autophosphorylation. The co-receptor BAK1 is activated by BRI1-dependent transphosphorylation, and BAK1 subsequently enhances signaling output through BRI1 and BAK1 transphosphorylation reciprocally (Wang et al. 2008).

\section{Tyrosine phosphorylation of plant proteins including receptor kinases}

Plant protein phosphorylation was always exciting and importance because it is involved in regulating just about everything such as cell cycle, metabolism, stress responses, pathogen responses, transport, hormones, light, and development. For examples, an alfalfa cyclin-dependent kinase inhibitor (CKI) that itself becomes phosphorylated by a calmodulin-like domain kinase (CDPK). Epitope tagging has shown that tobacco NtCDPK2 is shared between pathway triggered by biotic and abiotic stresses, pathogen responses to MAP kinase signaling, peptide elicitor PEP-13 in parsely, which is stimulates rapid $\mathrm{Ca}^{2+}$ influx that sustains $\mathrm{Ca}^{2+}$ levels required for ROS production and activation of three MAPKCs. Phosphorylation by a cell wall-associated kinase (WAK) inactivates the tobacco mosaic virus movement protein (TMV-MP) required for vial genome transport between cells (Mundy and Schneitz, 2002). As another interesting data, differential phosphorylation of import receptors in the chloroplast outer envelope complex (Toc) regulate chloroplast preprotein import. Solid-phase renaturation assays indicate that outer envelope protein phosphorylate the Toc34 and Toc159 receptors. In light signaling, histone kinase activity in Arabidopsis is induced by high irradiance levels during de-etiolation. The kinase that phosphorylates the COP9 signalosome has been partially purified (Mundy and Schneitz, 2002).

Huang (2003) has been reported that soluble protein phosphorylated on tyrosine residue(s) during phytohormone-stimulated hypocotyl cell proliferation in Arabidopsis thaliana. Although a few functional information is known for most of LRR-RLKs, members of the plant LRR-RLK family are very highly conserved cytoplasmic domains that shown to phosphorylate serine and threonine residues, and also phosphorylate tyrosine residues (Chang et al. 1992; Goring and Rothstein 1992; Mu et al. 1994; Shiu and Bleecker 2001). Although phosphorylation of a $\beta$ tubulins dimers by protein tyrosine kinases plays an importance functional role in the regulation of cellular growth and differentiation in animal cells system, in plant, however, the functional role of tubulin protein tyrosine phosphorylation is still unclear. Blume et al. (2008) studied that tubulin isolated by both immunoprecipitation with antibody and DEAE-chromatography method is phosphorylated on tyrosine residues in suspension cultured cells of N. tabacum. Interestingly, Fedina et al. (2008) reported that effect of BR on tyrosine phosphorylation of the Calvin cyclerelated enzymes. As we known, activities of Rubisco and other Calvin cycle enzymes can be controlled through phytohormoneinduced changes in their phosphorylation. They used 2D-SDSPAGE was used to separate proteins from total proteins of pea (Pisum sativum L.) leaves, and then total soluble proteins characterized with Western blot hybridization using specific antibodies. This analysis shown that over 40 proteins were phosphorylated on tyrosine residues. Some of these proteins were significantly changed under the action of BR. Also, it induces a large range of cell responses including plant growth, seed germination, and nitrogen fixation; it also improves plant resistance to biotic and abiotic stresses such as cold, pathogen, and salt 
stress (Kulaeva et al. 1989). Also, we fractionated total soluble and membrane proteins with ultracentrifugation (31,000 rpm, 2 hrs) from Arabidopsis thaliana tissues, and the total proteins were subjected to Western blot hybridization with phospho-specific antibodies such as phosphotyrosine and phosphothreonine as shown at Figure 1. According to our data, many proteins are phosphorylated by BR treatment. Generally, tyrosine phosphorylation in plants is investigated to lesser expand than phosphorylation on serine and threonine residues. Nevertheless, substantial evidence has accumulated on the involvement of this phosphorylation in the regulation of plant metabolism. Interestingly, many Serine and Threonine phosphorylation sites have been identified on both BRI1 and BAK1 (Oh et al. 2000; Wang et al. $2005 ; 2008)$. As an interesting result, Oh et al. (2009a) reported that $\mathrm{BR}$ receptor, BRI1 receptor kinase was phosphorylated on tyrosine residues in vivo which are Tyr-831 and Tyr-956. As interesting results, BRI1 (Y831F)-Flag are larger than wild-type BRI1-Flag (Oh et al. 2009b), which suggested that phosphorylation of Tyr-831 inhibits plant growth (Fig. 2A). Oh et al (2011) characterized BRI1 (Y831F)-Flag transgenic plants in terms of leaf shape and relative growth rate (RGR), photosynthetic rate, and specific gene expression to understand the actual mechanism for the increased growth. Overall, RGR was increased $16 \%$ in the BRI1(Y831F)-Flag plants, and the gain of function of the BRI1(Y831F)-Flag was dominant in the wild-type background, these results suggested that phenotype of BRI1(Y831F)-Flag transgenic plants are consistent in both bril-5 and wild type

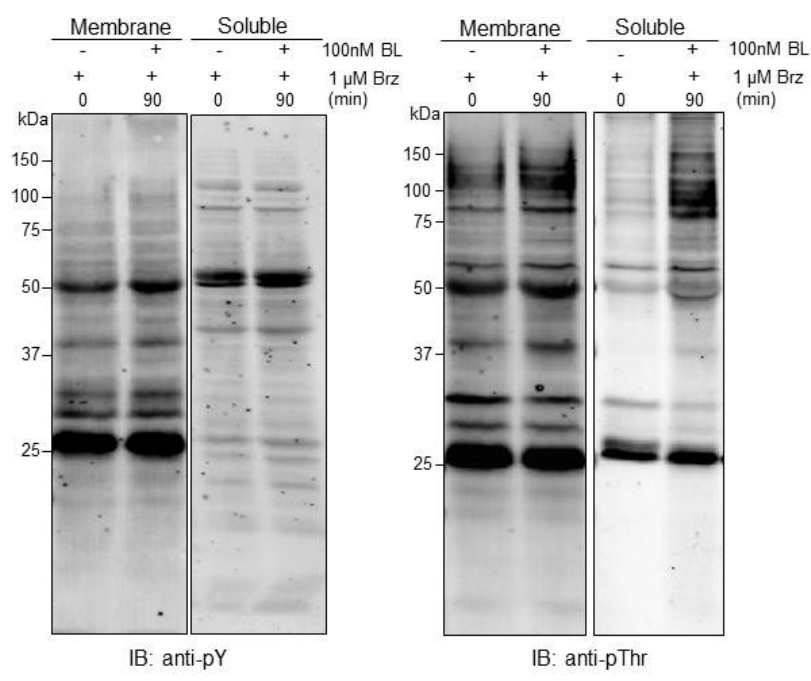

Fig. 1 Total soluble and membrane protein tyrosine and threonine phosphorylation patterns in the absence and presence of brassinolide $(100 \mathrm{nM})$. A. thaliana seedlings were grown in shaking liquid culture and microsomal membranes were isolated. Total soluble and membrane proteins were extracted and clarified by centrifugation. Proteins $(30 \mu \mathrm{g} /$ well) were separated by $10 \%$ SDS-PAGE, followed by transfer to PVDF membranes and Western blot analysis performed using anti-pY (1:500 dilution) and anti-pThr (1:500 dilution). Molecular weight markers are indicated in the left lane. The results shown are representative two separate experiments background. As another aspects, microarray analysis indicated that specific genes expression profile associated with cell wall biosynthesis, vascular differentiation and brassinolide biosynthesis were altered highly in gene expression and may significantly contribute to the changes in entire plant growth. In terms of analysis of chlorophyll fluorescence and gas exchange, Oh et al., (2011) demonstrated that BRI1(Y831F)-Flag transgenic plant had about $20 \%$ higher photosynthetic rate, and metabolite analysis documented enhanced accumulation of carbohydrates and most prominently glycine and proline among free amino acids. Taken together, these results suggested that mutation of tyrosine phosphorylation site of BRI1 receptor kinase can enhance photosynthetic rate and leaf growth. This is an excellent example
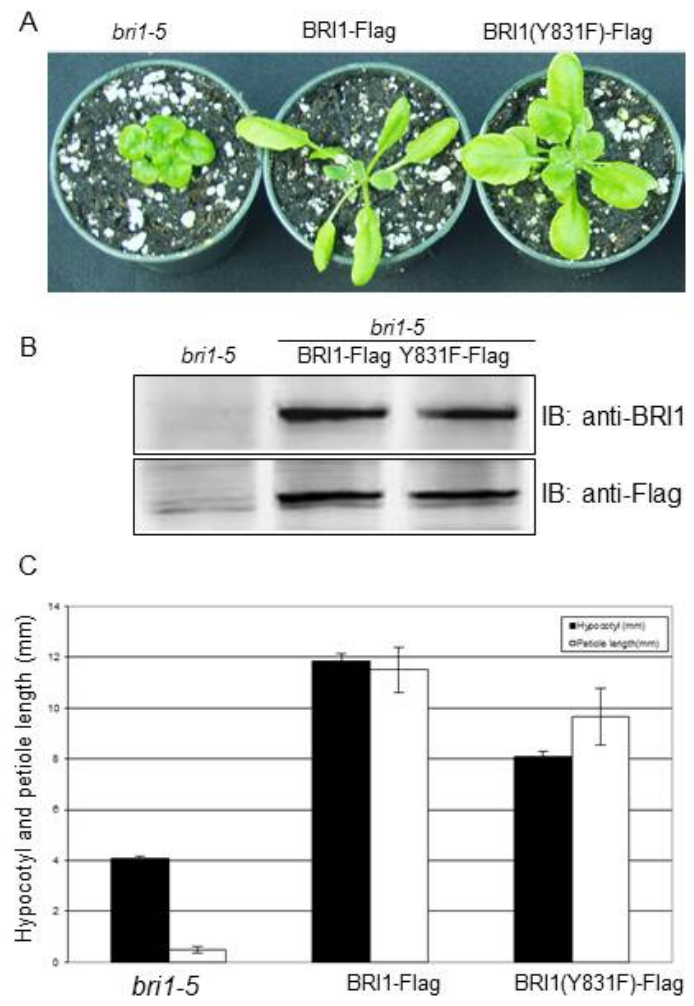

Fig. 2 Comparison of BRI1 (Y831F)-Flag and wild type BRI1-Flag in the bri1-5 mutant (A-C). (A) Thirty-three-day-old plants (bril-5 background) grown in soil ( $8 \mathrm{hr}$ of light/16hr of dark). (B) Protein expression levels of transgenic plants on BRI1-Flag and BRI1 (Y831F)-Flag in the bri1-5 backgrounds. To confirm protein level of BRI1-Flag and BRI1 (Y831F)-Flag, A. thaliana plants were grown in soil and microsomal membranes were isolated. Membranes were solubilized with Triton X-100 and clarified by centrifugation. Immunoprecipitated BRI1-Flag and BRI1-Flag (Y831F)-Flag proteins were subjected to SDS-PAGE, followed by transfer to PVDF membranes and Western blot analysis performed using anti-BRI1 (1:1000 dilution) and anti-Flag antibodies (1:1000 dilution). (C) Altered hypocotyl and petiole length of transgenic plants expressing BRI1 (Y831F)-Flag and BRI1-Flag. Seedlings of bri1-5, wild type BRI1-Flag and BRI1 (Y831F)-Flag were grown for six days in the dark. Seedlings were grown on vertical plates $(1.2 \%$ agar, $1 \%$ sucrose $)$ with half-strength MS media 
where a specific tyrosine residue of plant receptor kinases has been shown to play an important functional role in vivo in plant receptor kinase and crop plant improvement (Fig. 2). As another interesting plant receptor kinase, EF-TU RECEPTOR (EFR) is activated depend on ligand binding by phosphorylation on its tyrosine residues. Phosphorylation of a tyrosine residue, Y836, is an essential for activation of EFR and downstream plant innate immunity to the Pseudomonas syringae (Macho et al. 2014).

\section{Dual-specificity of receptor kinases}

Plant LRR-RLKs are classified as Ser/Thr kinases in contrast to animal RTK such as the epidermal growth factor family, although a few reports suggested that LRR-RLKs might be dual functional kinases (Albrecht et al. 2008; Rudrabhatla et al. 2006; Sugiyama et al. 2008). During our extensive screen of in vitro LRR-RLK phosphorylation sites, we found many LRR-RLKs that indeed autophosphorylated on Tyr, in addition to Ser and Thr. This led to a detailed analysis of the functional role of tyrosine phosphorylation in BR signal transduction pathway. Two BRIl tyrosine phosphorylation sites, Y831 and Y956 were identified with customized phosphospecific peptide antibodies in vitro and in vivo. Tyr-831 in the JM domain of BRI1 has a regulatory role, with phosphorylation of Tyr-831 leading inhibition of plant growth and control of flowering time. In contrast, Tyr-956 is located in kinase domain and is critical for BRI1 receptor kinase activity. Transgenic plants of BRI1 (Y831F)-Flag in the bri1-5 mutant recovered the dwarf phenotype (Oh et al. 2009b). This was the first demonstration of in vivo function of Tyr phosphorylation in an LRR-RLK.

Also, Oh et al.(2010) identified Tyr-610 in the carboxy terminal domain of BAK1 as a major site of autophosphorylation in vitro and in vivo. In case of BAK1, it is established as a dual-specificity receptor kinase. The site-directed mutagenic analysis also identified Tyr-463 as essential for kinase activity, as the BAK1 (Y463F) site-directed mutant was inactive. Karlova et al. (2009) identified the autophosphorylation site in corresponding tyrosine residue of SERK1 in vitro. However, it still remains to know whether autophosphorylation of this Tyr residue is critical for kinase activity. Recently, as another interesting report, tyrosine phosphorylation regulates the activity of phytochrome $\mathrm{B}$ photoreceptor (Nito et al. 2013). They examined whether phytochrome B is phosphorylated in response to light, and eventually, $\mathrm{N}$-terminus of this photoreceptor contains a region with a number of serines, threonines, and tyrosines. Interestingly, phosphorylation of tyrosine104 residue appears to play a negative role in phyB's activity with light-dependent manner (Nito et al. 2013).

However, currently how tyrosine phosphorylation of downstream signaling components might be affected by BR, light, pathogens and other ligands certainly appears as an very exciting aspect to pursue in the near future studies.

\section{Possible role of tyrosine phosphorylation in Arabidopsis thaliana}

Current results are many essential questions for tyrosine phosphorylation that still unclear to be answered. Identifying the receptor kinase domain motifs of the LRR-RLKs that control the ability to autophosphorylate on Tyrosine residue(s) will be an exciting question to explore in the future. Another interesting questions will be focusing on the molecular and signaling events responsible for significantly increased plant growth and flowering time of BRI1(Y831F)-Flag transgenic plants compared to BRI1-Flag transgenic plants. Although good evidence was presented by $\mathrm{Oh}$ et al. (2011), the molecular understanding of how Tyr 831 autophosphorylation affects plant growth an flowering time, still unclear to be elucidated. To understand these importance questions, Oh et al. (2011) has been performed a transcriptome analysis of the BRI1(Y831)-Flag and BRI1-Flag. As predicted from the divergent growth phenotypes of BRI1-Flag versus BRI1(Y831F)Flag, the many gene expression profile is highly altered suggesting that basic signaling mediated by BRI1 altered when Tyr- 831 is in the phosphorylated versus unphosphorylated state (Fig. 3). Phosphorylation could direct affect the conformation of the JM domain or could mediate the interaction with one or more tyrosine phosphorylation-binding proteins (see Fig. 3), which

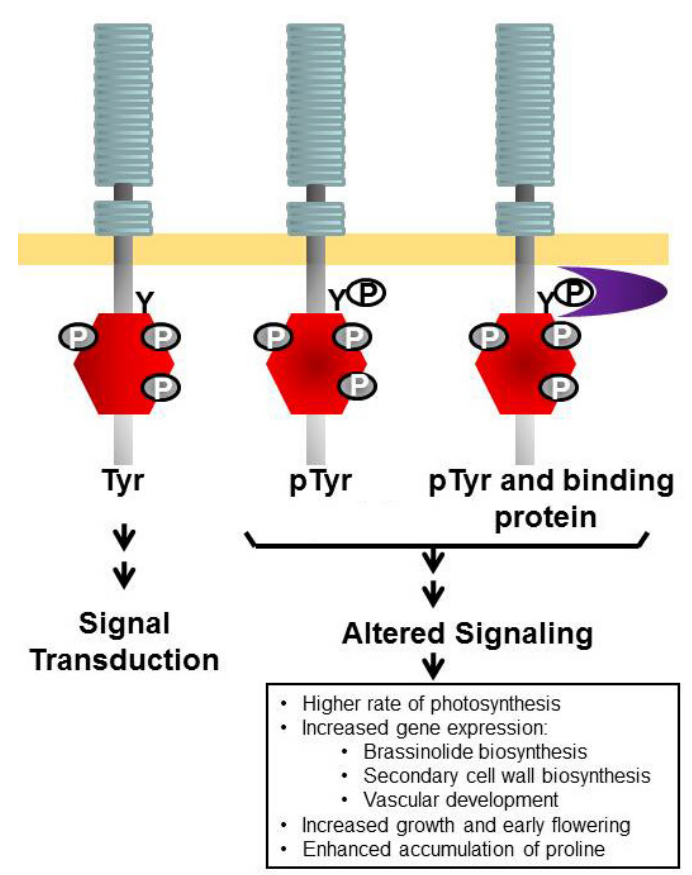

Fig. 3 Working model for the effect of Tyr-831 phosphorylation on BRI1-mediated signal transduction. Site-directed mutagenic analysis suggests that tyrosine phosphorylation of the Y831 site in the BRI1 cytoplasmic domain affects BR signal transduction. This alteration in signaling may be a direct result of tyrosine phosphorylation, possibly including a conformational change, or may involve an unknown phosphotyrosine-binding protein. This figure is adapted from Oh et al. (2009b) and modified 
have not been investigated in plants but are well known to play an critical role in signal transduction pathway in animal systems (Yaffe, 2002).

\section{Conclusions}

Protein phosphorylation in higher plants including Arabidopsis thaliana predominantly occurs on serine (Ser) and threonine (Thr) residues, whereas phosphorylation on tyrosine (Tyr) residues is less abundant (Bentem and Hirt, 2009). However, as we know, mass-spectrometry-based analyses and phosphotyrosine-specific antibodies indicate that Tyr phosphorylation is as extensive in plants as it is in animals. We have shown that many plant proteins including receptor kinases are autophosphorylated and transphosphorylated on tyrosine residues (Oh et al., 2009; 2010; 2012), As described in the Introduction, tyrosine phosphorylation in BR receptor, BRI1 is unexpected and surprising because BRI1 has only been shown in autophosphorylated on serine and threonine residues (Oh et al. 2000) as previous data. As another example, PRK1 receptor kinase that was shown to autophosphorylate on serine and tyrosine residues in vitro ( $\mathrm{Mu}$ et al. 1994), and AtSERK1 was reported to phosphorylate myelin basic protein and casein on serine, threonine, and tyrosine residues in vitro (Shah et al. 2001). Both results indicated that PRK1 and AtSERK1 are dual-specificity receptor kinases. In addition BRI1 and BAK1, many recognized receptor kinases, including BKK1 (He et al. 2007), HAESA (Jinn et al. 2000), and the lightrepressible receptor kinase (Deeken and Kaldenhoff, 1997) also has dual-specificity kinase activity. Based on this cumulative data we now propose a proteomic screen of Tyr phosphorylation sites across the LRR-RLK family. After identification of Tyr phosphorylation sites of many diverse receptor kinases, we will functionally characterize the role of phosphorTyr in selected LRR-RLKs. The phosphorylated tyrosine residues will be substituted with Phe to eliminate phosphorylation and with Asp to mimic constitutive phosphorylation, as we have done for BRI1 and BAK1 (Wang et al. 2005; 2008). In terms of protein biochemical studies, PTMs can be extended by altering the residue modified by directed mutagenesis to mimic the modified state or to prevent the modification. We believe that these approaches are useful to not only characterize the regulatory role of a given modification, but may also provide opportunities for plant improvement. The example involves the receptor kinase, BRI1 that is required for brassinosteroid signaling. BRI1, and its co-receptor kinase, BAK1, initiate the signaling pathway that regulates the expression of numerous genes in the nucleus and enhances growth and yield, as well as stress tolerance. The receptor kinases are regulated by phosphorylation on numerous sites, some of which such as Tyr-831, are 'inhibitory autophosphorylation' sites that we postulate function to attenuate signaling when modified. Studies with the BRI1 (Y831F) directed mutant suggest that removing this modification can protract signaling and thereby enhance plant growth, providing the example where manipulation of a PTM has potential for plant improvement.

\section{Acknowledgements}

This work was supported by Research Fund of Chungnam National University (CNU), Daejeon, Korea.

\section{References}

Albrecht C, Russinova E, Kemmerling B, Kwaaitaal M, and de Vries SC (2008) Arabidopsis SOMATIC EMBRYOGENESIS RECEPTOR KINASE proteins serve brassinosteroid-Dependent and -independent signaling pathways. Plant Physiol 148:611-619

Becraft PW (2002) Receptor kinase signaling in plant development. Annu Rev Cell Dev Biol 18:163-192

Bentem SF, Hirt H (2009) Protein tyrosine phosphorylation in plants: more abundant than expected? Trends in Plant Science 14:71-76

Blume Y, Yemets A, Sulimenko V, Sulimenko T, Chan J, Lloyd C, Draber P (2008) Tyrosine phosphorylation of plant tubulin. Planta 229:143-150

Boller T, Felix, G (2009) A renaissance of elicitors: perception of microbe-associated molecular patterns and danger signals by pattern-recognition receptors. Annu Rev Plant Biol 60:379-406

Cock JM, Vanoosthuyse V, Gaude, T (2002) Receptor kinase signalling in plants and animals: distinct molecular systems with mechanistic similarities. Curr Opin Cell Biol, 14:230-236

Cho SK, Larue CT, Chevalier D, Wang H, Jinn TL, Zhang S, Walker JC (2008) Regulation of floral organ abscission in Arabidopsis thaliana. Proc Natl Acad Sci USA 105:15629-15634

Chang C, Schaller GE, Patterson SE, Kwok SF, Meyerowitz EM, Bleecker AB (1992) The TMK1 gene from Arabidopsis codes for a protein with structural and biochemical characteristics of a receptor protein kinase. Plant Cell 4:1263-1271

Clouse SD (2011) Brassinosteroid signal transduction: From receptor kinase activation to transcriptional networks regulating plant development. Plant Cell 23:1219-1230

Clouse SD (2002) Brassinosteroid signal transduction: clarifying the pathway from ligand perception to gene expression. Mol Cell 10:973-982

Clouse SD, Sasse JM (1998) Brassinosteroids: Essential regulators of plant growth and development. Annu Rev Plant Physiol Plant Mol Bio 49:427-451.

de Lorenzo L, Merchan F, Laporte P, Thompson R, Clarke J, Sousa C, Crespi M (2009) A novel plant leucine-rich repeat receptor kinase regulates the response of Medicago truncatula roots to salt stress. Plant Cell 21:668-680

DeYoung BJ, Bickle KL, Schrage KJ, Muskett P, Patel K, Clark SE (2006) The CLAVATA1-related BAM1, BAM2 and BAM3 receptor kinase-like proteins are required for meristem function 
in Arabidopsis. Plant J 45:1-16

Deeken R, Kaldenhoff R (1997) Light-repressible receptor protein kinase: A novel photo-regulated gene from Arabidopsis thaliana. Planta 202:479-486

Fedina E, Karimova F, Tarchevsky I, Toropygin I, Khripach V. Effect of epibrassinolide on tyrosine phosphorylation of the calvin cycle enzymes. Russ J Plant Physiol 2008; 55:193-200

Gendron JM, Wang ZY (2007) Multiple mechanisms modulate brassinosteroid signaling. Curr Opin Plant Biol 10:436-441

Going DR, Rothstein SJ (1992) The S-locus receptor kinase gene in a self-incompatible Brassica napus line encodes a functional serine/threonine kinase. Plant Cell 4:1273-1281

Heldin, C (1995) Dimerization of cell surface receptors in signal transduction. Cell 80:213-224

He K, Gou X, Yuan T, Lin H, Asami T, Yoshida S, Russell SD, Li J (2007) BAK1 and BKK1 regulate brassinosteroid-dependent growth and brassinosteroid-independent cell-death pathways. Curr Biol 17:1109-1115

Huang H-J, Lin Y-M, Huang D-D, Takuya Takahashi, Sugiyama M(2003) Protein tyrosine phosphorylation during phytohormonestimulated cell proliferation in Arabidopsis hypocotyls. Plant Cell Physiol 44:770-775

Jinn TL, Stone JM,Walker JC (2000) HAESA, an Arabidopsis leucine-rich repeat receptor kinase, controls floral organ abscission. Genes Dev 14:108-117

Karlova R, Boeren S, van Dongen W, Kwaaitaal M, Aker J, Vervoort J, de Vries S (2009) Identification of in vitro phosphorylation sites in the Arabidopsis thaliana somatic embryogenesis receptor-like kinases. Proteomics 9:368-379

Li J, Nam KH (2002) Regulation of brassinosteroid signaling by a GSK3/SHAGGY-like kinase. Science 2002; 295:1299-1301

Li J, Wen J, Lease KA, Doke JT, Tax FE, Walker JC (2002) BAK1, an Arabidopsis LRR receptor-like protein kinase, interacts with BRI1 and modulates brassinosteroid signaling. Cell 110: 213-222

Matsubayashi Y, Sakagami Y (2006) Peptide hormones in plants. Annu Rev Plant Biol 57:649-674

Kulaeva ON, Burakhanova EA, Fedina AB (1989) Brassinosteroids regulate protein synthesis in wheat leaves. Dokl Akad Nauk SSSR 305:1277-1279

Mundy J, Schneitz K (2002) Protein phosphorylation in and around signal transduction. Trends in Plant Science 7:54-55

Macho AP, Schwessinger B, Ntoukakis V, Brutus A, Segonzac C, Roy S, Kadota Y, Oh M-H, Sklenar J, Derbyshire P, LozanoDurán R, Malinovsky FG, Monaghan J, Menke FL, Huber SC, He SY, Zipfel C (2014) A Bacterial tyrosine phosphatase inhibits plant pattern recognition receptor activation. Science 343:1509-1512

Mu JH, Lee HS, Kao TH (1994) Characterization of a pollenexpressed receptor-like kinase gene of Petunia inflate and the activity of its encoded kinase. Plant Cell 6:709-721

Nito K, Wong C, Yates III J, Chory J (2013) Tyrosine phosphorylation regulates the activity of phytochrome photoreceptors. Cell Reports 3:1970-1979

Nodine MD, Yadegari R, Tax FE (2007) RPK1 and TOAD2 are two receptor-like kinases redundantly required for arabidopsis embryonic pattern formation. Dev Cell 12:943-956

Clay NK, Nelson T (2002) VH1, a provascular cell-specific receptor kinase that influences leaf cell patterns in Arabidopsis. Plant Cell 14:2707-2722.

Oh MH, Wang X, Kota U, Goshe MB, Clouse SD, Huber SC (2009a) Tyrosine phosphorylation of the BRI1 receptor kinase emerges as a component of brassinosteroid signaling in Arabidopsis. Proc Natl Acad Sci USA 106:658-663

Oh MH, Clouse SD, Huber SC (2009) Tyrosine phosphorylation in brassinosteroid signaling. Plant Signaling \& Behav 4:1182-1185

Oh MH, Wang X, Wu X, Zhao Y, Clouse SD, Huber SC (2010) Autophosphorylation of Tyr-610 in the receptor kinase BAK1 plays a role in brassinosteroid signaling and basal defense gene expression. Proc Natl Acad Sci USA 107:17827-17832

Oh MH, Wu X, Clouse SD, Huber SC (2011) Functional importance of BAK1 tyrosine phosphorylation in vivo. Plant Signal and Behav 63:400-405

Oh MH, Ray WK, Huber SC, Asara JM, Gage DA, Clouse SD (2000) Recombinant brassinosteroid insensitive 1 receptor-like kinase autophosphorylates on serine and threonine residues and phosphorylates a conserved peptide motif in vitro. Plant Physiol 124:751-766

Oh MH, Wu X, Kim SK, Harper JF, Zielinski RE, Clouse SD, and Steven C. Huber (2012) CDPKs are dual-specificity protein kinases and tyrosine autophosphorylation attenuates kinase activity. FEBS Letter 586:4070-4075

Rudrabhatla P, Reddy MM, Rajasekharan R (2006) Genome-wide analysis and experimentation of plant serine/ threonine/ tyrosine-specific protein kinases. Plant Mol Biol 60:293-319.

Schlessinger J (2000) Cell signaling by receptor tyrosine kinases. Cell 103:211-225

Shiu SH, Bleecker AB (2001a) Receptor-like kinases from Arabidopsis form a monophyletic gene family related to animal receptor kinases.

Shiu SH, Bleecker AB (2001b) Plant receptor-like kinase gene family: Diversity, function and signaling. Sci STKE 113:re22

Shah K, Vervoort J, de Vries SC (2001) Role of threonine in the Arabidopsis thaliana somatic embryogenesis receptor kinase 1 activation loop in phosphorylation. J Biol Chem 276:41263-41269

Shah K, de Vries SC (2001) Role of threonine in the Arabidopsis thaliana somatic embryogenesis receptor kinase 1 activation loop in phosphorylation. J Bio Chem 276:41263-41269

Sugiyama N, Nakagami H, Mochida K, Daudi A, Tomita M, Shirasu K, Ishihama, Y (2008) Large-scale phosphorylation mapping reveals the extent of tyrosine phosphorylation in Arabidopsis. Mol Syst Biol, 4:193

Tor M, Lotze MT, Holton N (2009) Receptor-mediated signalling in plants: molecular patterns and programmes. J Exp Bot 60:3645-3654.

Torii KU (2004) Leucine-rich repeat receptor kinases in plants: structure, function, and signal transduction pathways. Int Rev Cytol 234:1-46

Vert, G, Nemhauser JL, Geldner N, Hong F, Chory J (2005) Molecular mechanisms of steroid hormone signaling in plants. 
Annu Rev Cell Dev Biol 21:177-201

Wang X, Goshe MB, Soderblom EJ, Phinney BS, Kuchar JA, Li J, Asami T, Yoshida S, Huber SC, Clouse SD (2005) Identification and functional analysis of in vivo phosphorylation sites of the Arabidopsis BRASSINOSTEROID-INSENSITIVE1 receptor kinase. Plant Cell 17:1685-1703

Yaffe MB (2002) Phosphotyrosine-binding domains in signal transduction. Nat Rev Mol Cell Biol 3:177-186

Wang X, Kota U, He K, Blackburn K, Li J, Goshe MB, Huber SC,
Clouse SD (2008) Sequential transphosphorylation of the BRI1/BAK1 receptor kinase complex impacts early events in brassinosteroid signaling. Dev Cell 15:220-235

Zhao D-Z, Wang Z-F, Speal B, Ma H (2002) The EXCESS MICROSPOROCYTES1 gene encodes a putative leucine-rich repeat receptor protein kinase that controls somatic and reproductive cell fates in the Arabidopsis anther. Genes \& Development 16:2021-2031 